\title{
A NUMERICAL APPROACH OF CHAOTIC MOTIONS IN A DUFFING-VAN DER POL OSCILLATOR
}

\author{
NGUYEN VAN DaO \\ Vietnam National University, Hanoi \\ Nguyen Van Dinh, Tran Kim Chi, Nguyen Dung \\ Vietnamese Academy of Science and Technology \\ (This paper has been published in: \\ International Conference on Nonlinear Analysis and Engineering \\ Mechanics today, $11^{\text {th }}-14^{\text {th }}$ Dec. 2006, Hochiminh city, Vietnam)
}

\begin{abstract}
In the present paper the influence of the excitation frequency $(\nu)$ and the forcing amplitude $(e)$ on the chaotic behaviour of the system governed by equation

$$
\ddot{x}+\omega^{2} x=k\left(1-\gamma x^{2}\right) \dot{x}+\beta x^{3}+e \sin \nu t
$$

will be examined. This equation is a Van der Pol one with a forcing term $e \sin v t$, where $\omega, \alpha, \gamma, e, \beta$ and $\nu$ are constants, overdot denotes the derivative with respect to time $t$. When $e=0, \alpha>0, \gamma>0$ we have the classical Van der Pol equation which represents a self-excited oscillator with the amplitude $a_{*}=2 / \sqrt{\gamma}$ and frequency $\omega$. Our discussion was focused upon variation of the excitation frequency $\nu$ and the forcing amplitude $e$. The bifurcation diagrams for acquiring the overview of equation $(0.1)$ and the Liapunov exponent method will be used $[3,4,5,6,9]$.

For a concrete case, the parameter regions in which either periodic or chaotic motions exist were shown. In two preceding cases, the first case, when $\nu$ is control parameter, it changes suddenly from periodic motion to chaotic motion, corresponding to Hopf bifurcation. In the second case, it is the double period process and leads to chaotic motion. Chaotic attractors illustrate the complexity of the motion of the system under consideration.
\end{abstract}

\section{SUMMARY OF THE CASE OF SMALL PARAMETERS}

First, we recall briefly some known results of deterministic motions in (0.1) for the case of smallness of the coefficients. It is supposed that $\nu$ is close to the natural frequency $\omega$, namely:

$$
\nu^{2}=\omega^{2}+\varepsilon \Delta,
$$

here $\Delta$ is a detuning parameter and $\varepsilon$ is a small positive one. Applying to (0.1) the asymptotic method [2] and using the amplitude and phase variables $(a, \theta)$ given by

$$
\begin{aligned}
& x=a \cos (\nu t+\theta), \\
& \dot{x}=-a \nu \sin (\nu t+\theta), \\
& \dot{a} \nu \cos (\nu t+\theta)-a \dot{\theta} \sin (\nu t+\theta)=0,
\end{aligned}
$$

we have

$$
\begin{aligned}
& \nu \dot{a}=-\varepsilon\left[\Delta x+k\left(1-\gamma x^{2}\right) \dot{x}+\beta x^{3}+e \sin \nu t\right] \sin (\nu t+\theta), \\
& \nu a \dot{\theta}=-\varepsilon\left[\Delta x+k\left(1-\gamma x^{2}\right) \dot{x}+\beta x^{3}+e \sin \nu t\right] \sin (\nu t+\theta) .
\end{aligned}
$$


Since $a$ and $\theta$ are slowly varying functions of time, the change in their values during a time period $T=2 \pi / \nu$ is very small. Hence, in the first approximation one may replace equations (1.3) by their time - averages over $(t, t+T)$ assuming $a$ and $\theta$ to be constant:

$$
\begin{aligned}
& \nu \dot{a}=\frac{\varepsilon}{2}\left[a k \nu\left(1-\frac{1}{4} \gamma a^{2}\right)-e \cos \theta\right], \\
& \nu a \dot{\theta}=\frac{\varepsilon}{2}\left(-\Delta a-\frac{3}{4} \beta a^{3}+e \cdot \sin \theta\right) .
\end{aligned}
$$

The steady-state equations are

$$
\begin{aligned}
& a_{0} k \nu\left(1-\frac{\gamma}{4} a_{0}^{2}\right)=e \cos \theta_{0}, \\
& \Delta a_{0}+\frac{3}{4} \beta a_{0}^{3}=e \sin \theta_{0} .
\end{aligned}
$$

By eliminating the phase $\theta_{0}$ from these equations we obtain

$$
A\left[(1-A)^{2}+\sigma^{2}\right]=E^{2},
$$

where

$$
A=\frac{\gamma}{4} a_{0}^{2}=\frac{a_{0}^{2}}{a_{*}^{2}}, E^{2}=\frac{\gamma}{4 k^{2} \omega^{2}} e^{2}, \sigma=\frac{\omega}{k}\left[\frac{\alpha^{2}-1}{\varepsilon}+\frac{3 \beta}{4 \omega^{2}} a_{0}^{2}\right], \alpha=\frac{\nu}{\omega},
$$

$a_{*}=2 / \sqrt{\gamma}$ is the amplitude of the purely self-excited Van der Pol oscillator. Below only the behaviour of forced oscillations with the frequency $\nu$ which is close to $\omega$ will be considered.

The oscillation described by the equation (0.1) with stationary amplitude $a_{0}$ and phase $\theta_{0}: x=a_{0} \cos \left(\nu t+\theta_{0}\right)$ has the frequency of the external force $\nu$ only. The self-excited oscillation is entrained by the external excitation. The synchronized oscillation (1.2) is characterized by the entrainment of the auto-periodic frequency by the external one. The synchronization effect is observed only when the exciting frequency $\nu$ is close enough to the natural frequency $\omega$.

The amplitude curves with various values of external excitation $(E)$ are given in the Fig. 1 for the case $\beta=0$ [1]. For $E=0$, i.e. for the zero external excitation, we find the results for the classical Van der Pol oscillator:

1) $A=0$ with $\sigma$ arbitrary,

2) $\sigma=0, A=1$.

Therefore, the resonance curves degenerate into the line $A=0$ ( $\sigma$-axis) and the point $\sigma=0, A=1$.

If $E$ is small but different from zero, we expect $A$ to be nearly 1 or nearly zero so that one of the response curves would be oval which is approximately the circle

$$
(A-1)^{2}+\sigma^{2}=E^{2}
$$

with centre at $\sigma=0, A=1$. In addition, the other branch runs near the $\sigma$-axis. The oval expands with increasing $E$. When $E$ increases, the resonance curves first consist of two branches, up to the critical value $E=2 / \sqrt{27}$ for which the two branches join at $\sigma=0$, $A=1 / 3$, then with further increase of $E$ the resonance curves have only a single branch.

From the Fig. 1 one can see that under certain conditions the frequency of the free oscillation is canceled out and is replaced by a synchronized oscillation, i.e. by an oscillation whose frequency is that of the external force, namely: 
1) For a given amplitude of the exciting force $(E)$, the synchronization effect is observed when the exciting frequency $\nu$ is close enough to the natural frequency $\omega$ of the oscillator. The larger the amplitude of the exciting force, the greater the frequency interval over which the synchronization occurs.

2) For a given exciting frequency, the oscillator is synchronized when the exciting amplitude is large enough. The closer the exciting frequency is to $\omega$, the lower its threshold amplitude is.

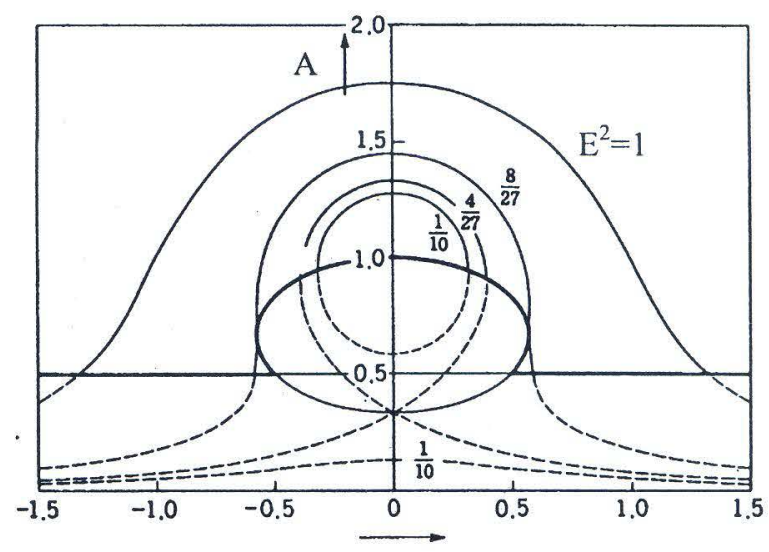

Fig. 1. Amplitude curves for the Duffing - Van der Pol oscillator $(\beta=0)$ with various values of external excitation

\section{THE CASE OF ARBITRARY PARAMETERS}

\subsection{Some concepts connected to bifurcation}

Bifurcation is a concept used to indicate a qualitative change in the features of a dynamical system, such as the number and type of solutions, under the variation of one or more parameters on which the considered system depends. These parameters are called the control parameters, and parameter values at which bifurcations occur are called bifurcation values. A bifurcation diagram is a graph of the state variables versus the parameters $[3$, $6,9]$.

The bifurcation diagram provides a summary of the essential dynamics and is therefore an important tool for examining the prechaotic or postchaotic changes in a dynamical system under parameter variations. The Poincaré map can be used to construct the bifurcation diagrams for continuous differential equations. When the data are sampled using a Poincaré map, it is very easy to observe period doubling and Hopf bifurcations. It is useful because one characteristic precursor to chaotic motion is the appearance of subharmonic periodic vibrations.

We'll examine two following concrete cases: a) The frequency $\nu$ is the control parameter, b) The forcing amplitude $e$ is the control parameter.

\subsection{The frequency $\nu$ is the control parameter}

We go back to the system (0.1) with $\omega^{2}=0.7, k=1, \gamma=0.6, \beta=-1, e=5$ and use the frequency $\nu$ as a control parameter. Poincaré sections for orbits of this system 
are constructed by using the excitation frequency $\nu$. For each orbit of the system the discrete points $(x(n T), \dot{x}(n T))$ are collected at time intervals of $T=2 \pi / \nu$ (the period of the external excitation force). The bifurcation diagram shown in Fig. 2 was generated by incrementing the control parameter $\nu$ in steps of $\Delta \nu=0.0001$. The graph consists of the points $(x(n T), \nu)$, where the values $x(n T)$ correspond to the attractor realized at each value of $\nu$.

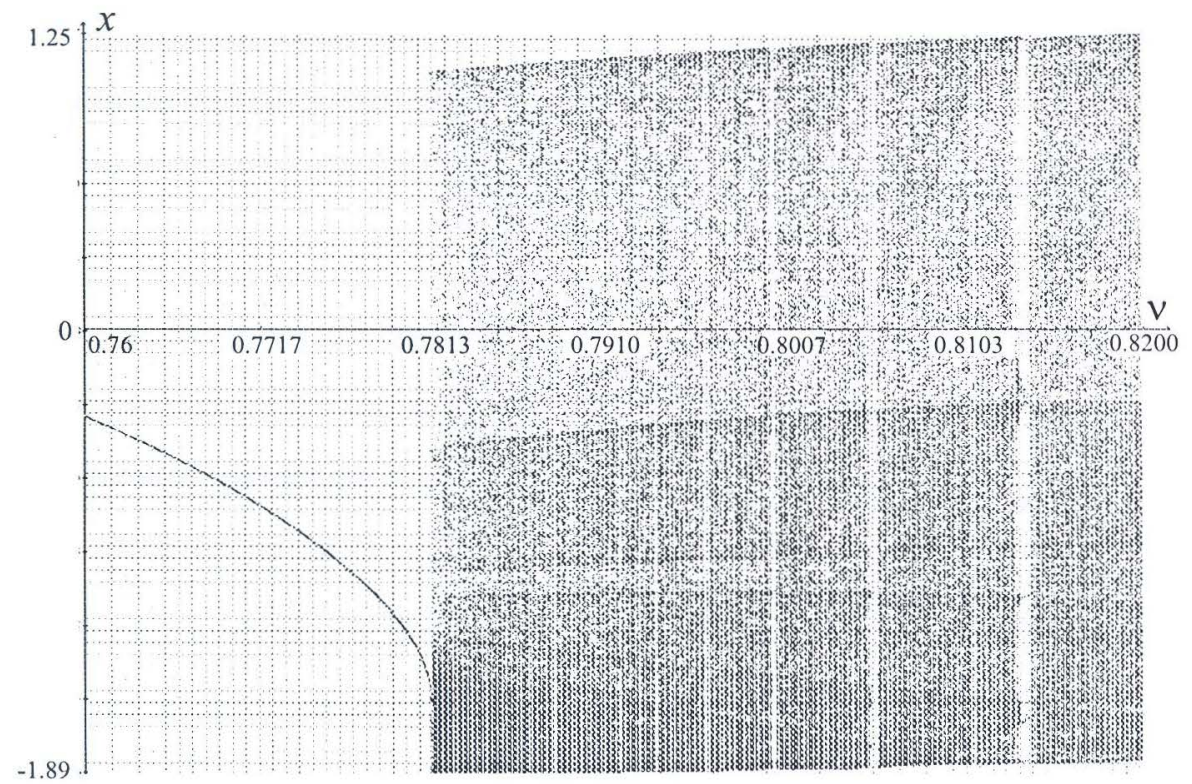

Fig. 2. Illustration of bifurcations on the poincarés section for $\omega^{2}=0.7, k=1, \gamma=0.6, \beta=-1, e=5$.

From Fig. 2 it is clear that as $\nu$ increased through $\nu_{h} \approx 0.780895$, there is an abrupt transition from the point attractor to an aperiodic one, so a Hopf bifurcation of a periodic solution (the Poincaré section consists of only one point) occurs. For $\nu<\nu_{h}$, the state of the system is periodic, when the control parameter exceeds the threshold value $\nu_{h}$, the system evolution is attracted to chaotic attractor, then the system undergoes a subcritical Hopf bifurcation. The attractors, both before and after the bifurcation, are shown in Fig. 3. Fig. 3a describes the periodic attractor with its Poincaré section consisting of one point (*) connected to $\nu=0.78$. With $\nu=0.782$ a chaotic motion occurs, Fig. 3b describes its attractor. We'll consider this case more detail below.

With the values of the frequencies $\nu<\nu_{h}$, the Poincaré sections consist of one point, the motions are periodic with the period equating the one of the external force. Beyond the periodic region occupying much of the interval $0.76 \leqslant \nu<\nu_{h}$, there exists a wide interval in which for certain ranges of the parameter $\nu$, the displacement $x$ takes an infinite number of values; these states are aperiodic.

It is also interesting to see that within the aperiodic regions there are narrow intervals in which the motion abruptly becomes periodic again, for example, the region around the value $\nu=0.8, \nu=0.8054, \nu=0.8138 \ldots$ In this interval, for every parameter $\nu, x$ takes a finite number of values (more than one), so that the corresponding motions are subharmonic oscillations. 


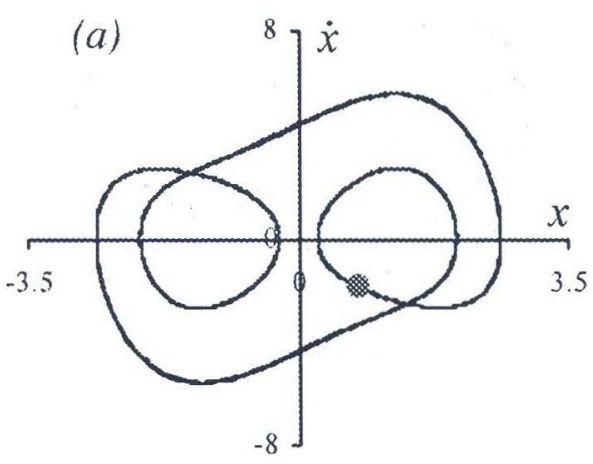

Fig. 3a. Periodic attactor $(\nu=0.78089)$

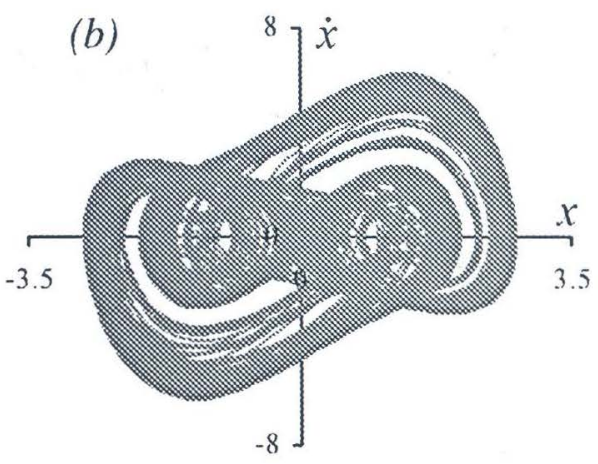

Fig. 3b. Chaostic attractor $(\nu=0.782)$

A concrete case of Chaotic motion. We consider a concrete case for the parameters $\omega^{2}=0.7, k=1, \gamma=0.6, \beta=-1, e=5$ and $\nu=0.782>\nu_{h}$. The aperiodic appearance of $x$ (see Fig. 4) suggests that the system under consideration is chaotic.

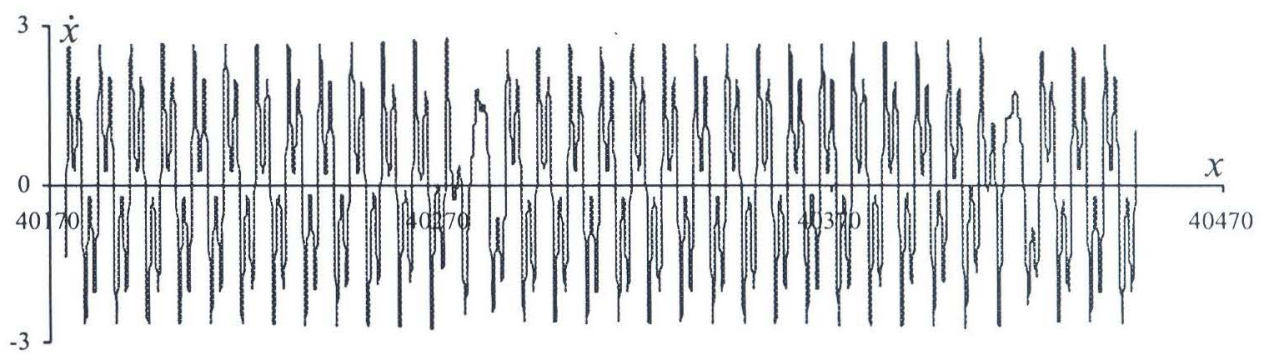

Fig. 4. Aperiode appearance of $x(t)(\nu=0.782)$

To verify that motion realized at $\nu=0.782$ is chaotic, we need to show the sensitivity to initial conditions on its attractor. We choose two points separated by $d_{0}=10^{-7}$ close to the attractor and examine initiated evalutions from them. Fig. 5 illustrates the variation of the separation $d$ with time $t$. The exponential growth of separation $d$ for $20<t<120$ is clearly noticeable. The separation saturates the size of the attractor for $t>120$. Therefore, there is a positive Liapunov exponent associated with the chaotic orbit at $\nu=0.782$ and its approximate value is $\lambda \approx 0.0495$. Much more insight can be gained from a Poincaré section (Fig. 6) consisting of stroboscopic points at instants $t=n(2 \pi / 0.782), n=0,1,2 \ldots$ of the orbit of the system $(2.1)$ in the space $(x, \dot{x})$. Fig. 6 shows the next 10000 points after the transition decays about the first 1000 periods. The corresponding attractor of the chaotic solution is presented in Fig. 3b. 


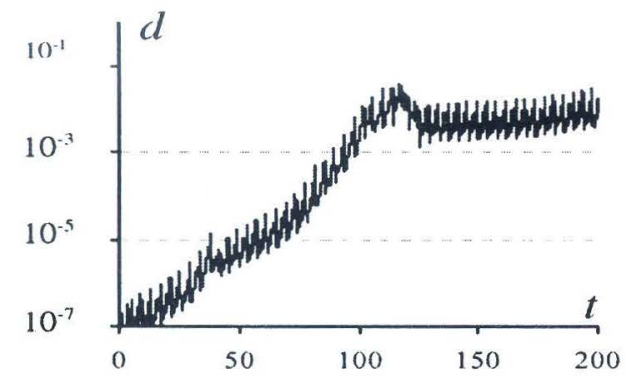

Fig. 5. Sensitivity to initial condition for $\nu=0.782$

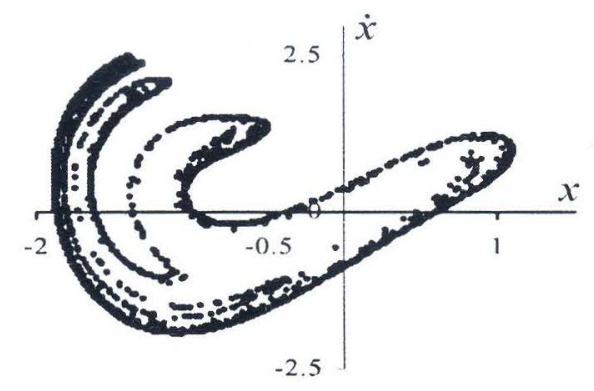

Fig. 6. Poincaré section for $\nu=0.782$

\subsection{The forcing amplitude $e$ is the control parameter}

We examine a graph of $x$ versus the forcing amplitude $e$ at $\omega^{2}=0.7, k=1, \gamma=0.6$, $\beta=-1, \nu=0.837$ in order to detect bifurcations. The bifurcation diagram is shown in Fig. 7. In this numerically constructed bifurcation diagram, the discrete points on the Poincaré section of the attractor realized at each value $e$ are displayed.

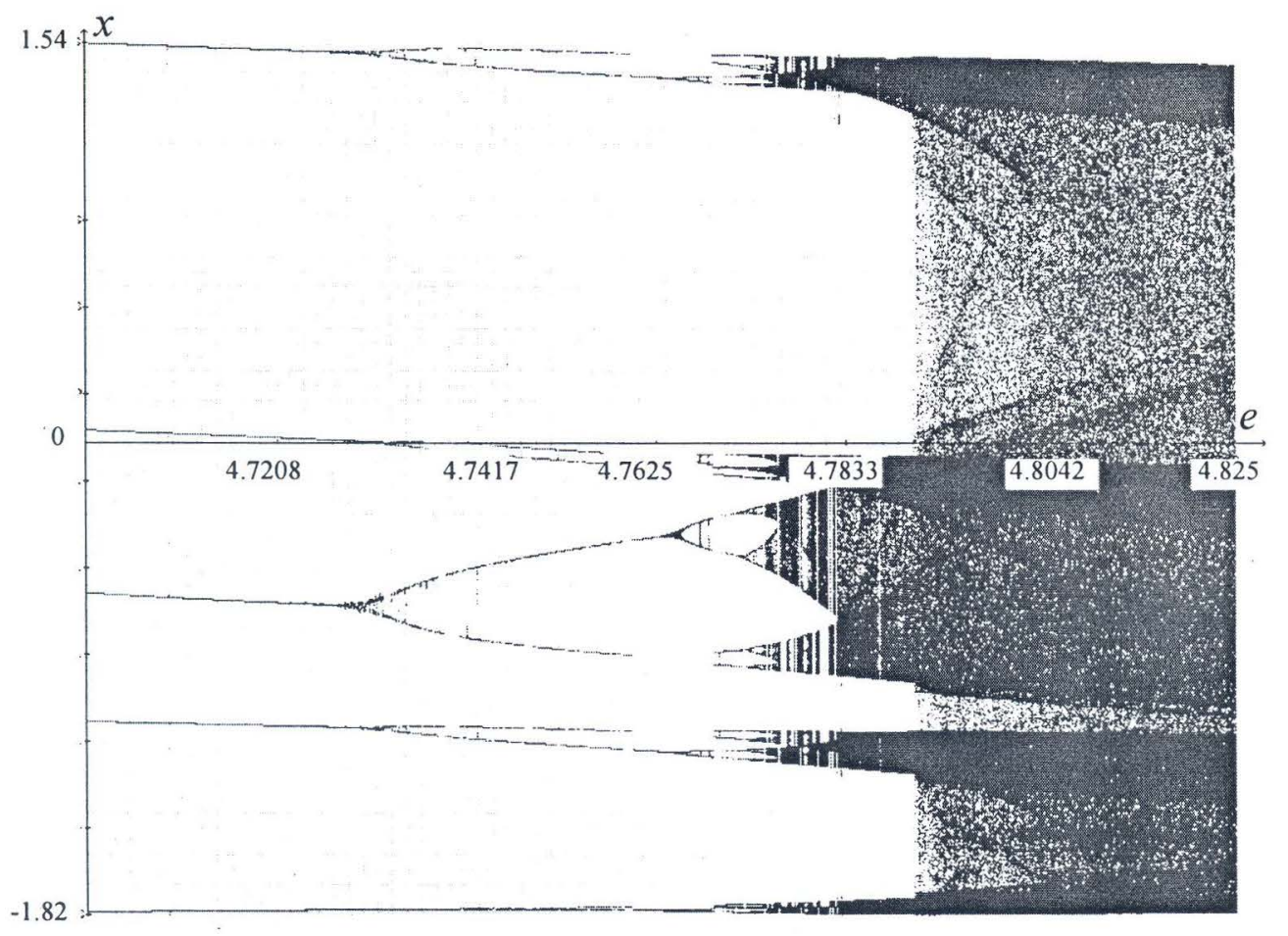

Fig. 7. Illustration of bifurcations on the Poincaé section

$$
\text { for } \omega^{2}=0.7, k=1, \gamma=0.6, \beta=-1, \nu=0.837
$$

Obviously, from Fig. 7, we can observe the sequence of period-doubling bifurcations. First, with one of values $e$ in the interval $(4.7,4.7645125)$, the Poincare section consists of five points (five dark points in Fig. 8), so there exist the subharmonic motions with the period equaling five times of the period of the external force (Fig. 8). At the value $e \approx 4.7645125$, the first period-doubling bifurcation occurs. After the bifurcation, with 
the values $e$ which is in the right vicinity of the value $e \approx 4.7645125$, the subharmonic motions with the period equaling twice the period of the previous motions appear, the Poincaré sections consist of ten points (Fig. 9), and so on. The chaotic attractor realized at $e=4.8042$ appearing after a sequence of period-doubling bifurcations is shown in Fig. 10, and Fig. 11 is corresponding attractor. The largest Liapunov exponent evaluated is positive $(\lambda \approx 0.0553)$ defines sensitivity to initial conditions on the chaotic attractor.

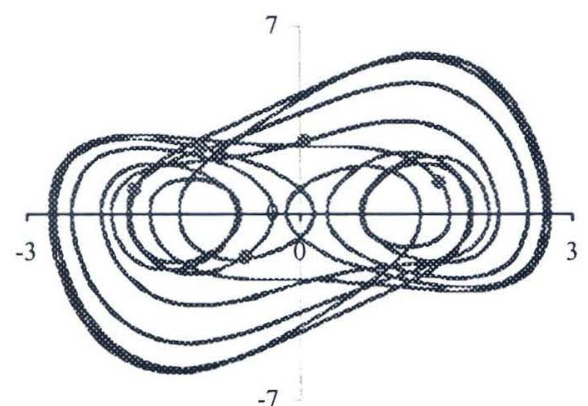

Fig. 8. Periodic orbit and its Poincré section (five points) for $e=4.7$

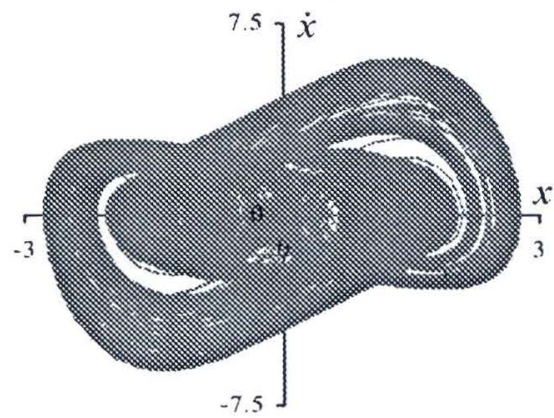

Fig. 10. Chaotic attractor for $e=4.8042$

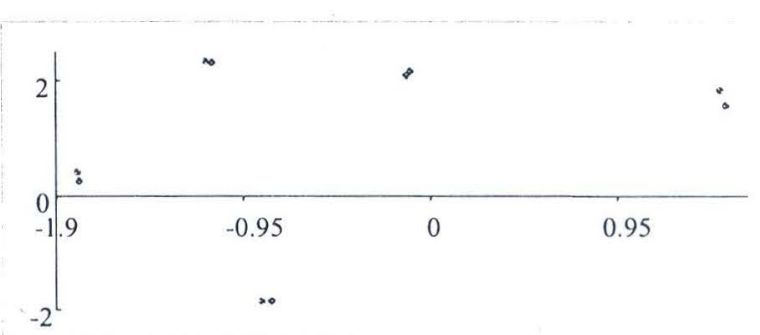

Fig. 9. Ten points in the Poincaré section for $e=4.7682$

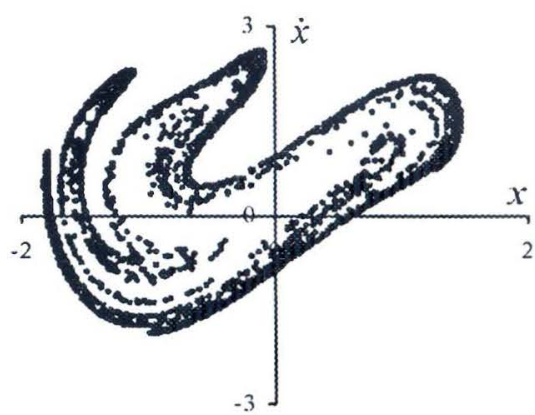

Fig. 11. Poincaré section for $e=4.8042$

The sequence of period-doubling bifurcations is one route to chaos and it is common in many dynamical systems. It is particularly interesting because it may be characterized by a certain universal constant (called the Feigenbaum's constant) that do not depend on nature of the concrete systems. This constant is considered as a specify criterion to determine if a system becomes chaotic by observing the prechaotic periodic behavior.

If the first bifurcation occurs at parameter value $e_{1}$, the second at $e_{2}$, and so on, then this constant is defined as

$$
\lim _{k \rightarrow \infty} \frac{e_{k}-e_{k-1}}{e_{k+1}-e_{k}}=\delta=4.6692016 \ldots
$$

Table 1 shows a list of the parameters at which period-doublings occur in the Poincaré map corresponding to the system (0.1) for $\omega^{2}=0.7, k=1, \gamma=0.6, \beta=-1, \nu=0.837$ and $e$ is used as the control parameter. 
Table 1. Feigenbaum's constant in the Poincaré map

\begin{tabular}{ccc}
\hline Period & Parameter $e$ & Ratio \\
\hline 5 & 4.7645125 & \\
10 & 4.7717130 & \\
20 & 4.77327499 & 4.600144 \\
40 & 4.773611 & 4.656677 \\
80 & 4.77368297 & 4.668751 \\
160 &. &.
\end{tabular}

Liapunov exponent. To determine the chaotic motion in the system described by (0.1) it is necessary to calculate the largest Lyapunov exponent. If $d_{0}$ is a measure of the initial distance between the two starting points, at a small but later time the distance is

$$
d(t)=d_{0} 2^{\lambda t} .
$$

The divergence of chaotic orbits can only be locally exponential, since if the system is bounded, as most physical experiments are, $d(t)$ cannot go to infinity. Thus, to define a measure of this divergence of orbits, we must average the exponential growth at many points along a trajectory. One begins with a reference trajectory and a point on a nearby trajectory and measures $d(t) / d_{0}$. When $d(t)$ becomes too large (i.e, the growth departs from exponential behavior), one looks for a new "nearby" trajectory and defines a new $d_{0}(t)$. One can define the first Lyapunov exponent by the expression

$$
\lambda=\frac{1}{t_{N}-t_{0}} \sum_{k=1}^{N} \log _{2} \frac{d\left(t_{k}\right)}{d_{0}\left(t_{k-1}\right)} .
$$

The motion is chaotic if the corresponding largest Lyapunov exponent is positive. For this calculation ([7]), in the case of concrete case of Chaotic motion $\omega^{2}=0.7, k=1, t=$ $0.6, \beta=-1, e=4.825$ and $v=0.837$, we represent the equation (0.1) in the form

$$
\begin{aligned}
& \dot{x}_{1}=x_{2}, \\
& \dot{x}_{2}=-0.7 x_{1}+\left(1-0.6 x_{1}^{2}\right) x_{2}-x_{1}^{3}+4.825 \sin (0.837 z), \\
& \dot{z}=1 .
\end{aligned}
$$

Let $u=\left(x_{1}, x_{2}, z\right)$ is a three dimension vector and $u^{*}=u^{*}\left(t, u_{0}\right)$ is a reference trajectory of the system (1.2), where $u_{0}$ is the initial condition. The variational equation corresponding to this reference trajectory is

$$
\dot{\eta}=A \eta,
$$

where $\eta=u-u^{*}$ and the matrix $A$ depends on $u^{*}$.

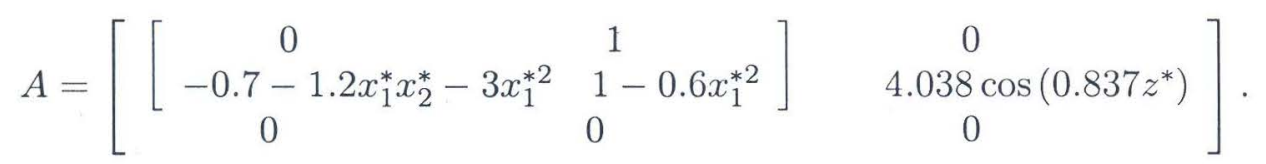

If this initial condition is chosen at random, then it is likely to have a component that lies in the direction of the largest positive eigenvalue of $\mathrm{A}$. It is the change in length in this direction that the largest Lyapunov exponent measures. 
After a given time interval $t_{k+1}-t_{k}=\tau$, take

$$
\frac{d\left(t_{k+1}\right)}{d\left(t_{k}\right)}=\frac{\left|\eta\left(\tau ; t_{k}\right)\right|}{\left|\eta\left(0 ; t_{k}\right)\right|} .
$$

The time evolution of the Lyapunov exponent is presented in Fig. 12. The largest Lyapunov exponent value is a positive number $\lambda \approx 0.0698>0$, which shows the chaotic character of the motion of the system (2.2). This means that two trajectories starting closely one to another in the phase space will move exponentially away from each other for small times on the average

$$
d(t)=d_{0} 2^{\lambda t},
$$

where $d_{0}$ is the initial distance between two adjacent starting points at $t=t_{0}$ and $d$ is the distance between two these points at the moment $t$. In Fig. 13, we show how the distance $d$ between evolutions initiated from two points separated by $d_{0}=10^{-7}$ varies with time. Both of the initial points are located close to the attractor. The separation clearly grows exponentially in the range $10<t<125$. The separation saturates at the size of the attractor for $t>125$.

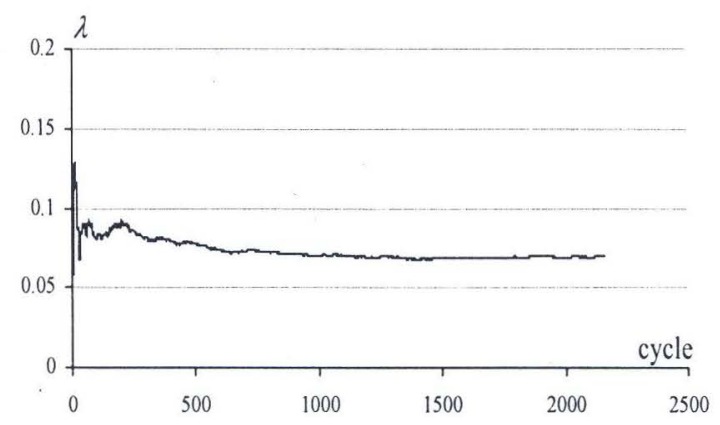

Fig. 12. Time evolution of the largest Liapunov exponent (one cycle $=2 \pi / \nu, \nu=0.837$ )

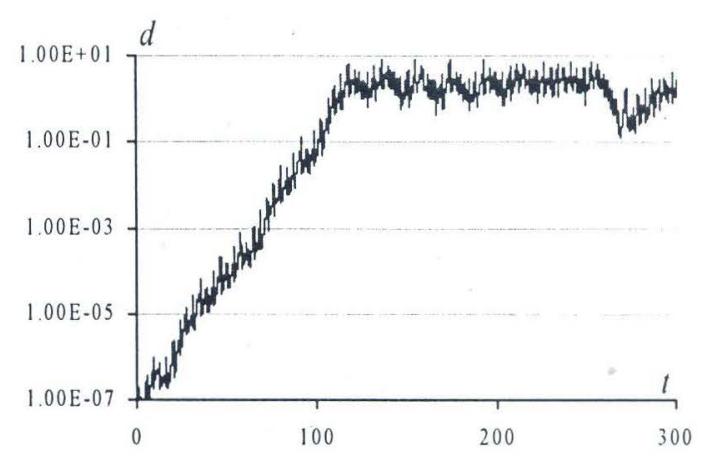

Fig. 13. Sensitivity to initial conditions on the attractor

\section{CONCLUSION}

The bifurcation diagrams and Liapunov exponent have been used for detecting chaotic regimes in the system described by equation (0.1). Our discussion was focused upon variation of the excitation frequency $\nu$ and the forcing amplitude $e$. For a concrete case, the parameter regions in which either periodic or chaotic motions exist were shown. Chaotic attractors (Fig. 5, 10) illustrate the complexity of the motion of the system under consideration. The bifurcation diagram shows so clearly the motions of system (0.1) with respect to the observed parameters. In two preceding cases, the first case, when $\nu$ is control parameter, it changes suddenly from periodic motion to chaotic motion, corresponding to Hopf bifurcation. In the second case, it is the double - period process and leads to chaotic motion. Lyapunov exponent value and some other criterions have used to determine chaotic motion of a solution. 


\section{REFERENCES}

1. Nguyen Van Dao, Stability of Dynamic Systems, Vietnam National University Publishing House, Hanoi, 1998.

2. Yu. A. Mitropolskii, Nguyen Van Dao, Applied Asymptotic Methods in Nonlinear Oscillations, Kluwer Publisher, 1997.

3. A. Nayfeh, Balakuma Balachandran, Applied Nonlinear Dynamics, John Wiley \& Sons, Inc., A WileyInterscience Publication, 1995.

4. T. Kapitaniak, W. H. Steeb, Transition to chaocs in a generalized Van der Pol's equation, Journal of Sound and Vibration 143 (1990) 167-170.

5. M. Lakshmanan, S. Rajasekar, Nonlinear Dynamics, Springer, 2003.

6. G. L. Baker, J. O. Gollub, Chaotic Dynamics. An introduction, Cambridge University Press, 1990.

7. Francis C Moon, Chaotic Vibration, John wiley, 1996.

8. T. Kathleen, Alligood, Tim D. Sauer, Jame A. Yorke. Chaos, An Introduction to Dynamical Systems, Springer Verlag, New york, Inc. 1997.

9. Thomas S. Parker, Leon O. Chua, Practical Nunerical Algorithms for Chaotic Systems, Springer Verlag, New york, Inc. 1989.

10. Nguyen Van Dao, Nguyen Van Dinh, Tran Kim Chi, Nguyen Dung, Chaotic motions in some nonlinear systems, The $5^{\text {th }}$ Asian Symposium on Applied Electromagnetics and Mechanics, Hanoi, October 10-14, 2005 .

\section{CHUYỄN DộNG HỖN DỘN TRONG CHẤN TƯ DUFFING - VAN DER POL}

Bài báo nghiên cứu ảnh hường của tần số và biên độ của lực kích động lên ứng xừ hỗn độn của một chấn tử mô tả bởi phương trình Duffing - Van der Pol:

$$
\ddot{x}+\omega^{2} x=k\left(1-\gamma x^{2}\right) \dot{x}+\beta x^{3}+e \sin \nu t
$$

Khi $e=0, \alpha>0, \gamma>0$ ta có một phương trình Van der Pol cổ điển biểu diễn cho một dao động tự kích với biên độ $a_{*}=2 / \sqrt{\gamma}$ và tần số $\omega$. Các tính toán số đã được thực hiện để nghiên cứu chuyển động hỗn độn xảy ra khi thay đổi tần số kích động $\nu$ và biên độ e. Các sơ đồ phân nhánh và phương pháp số mũ Liapunov đã được sừ dụng để nghiên cứu các trạng thái chuyển tiếp từ chuyển động tuần hoàn sang chuyễn động hỗn độn khi $\nu$ thay đổi (ứng với một dạng phân nhánh Hopf) và khi $e$ thay đổi (ứng với một quá trình nhân đôi chu kỳ trong sơ đồ phân nhánh). 